\title{
CRISPR/Cas9 gene editing uncovers the role of CTR1 and ROS1 in melon
} fruit ripening and epigenetic regulation

Andrea Giordano ${ }^{1 *}$, Miguel Santo Domingo ${ }^{1}$, Leandro Quadrana ${ }^{3}$, Marta Pujol ${ }^{1,2}$, Ana Montserrat Martín-Hernández ${ }^{1,2}$ and Jordi Garcia-Mas ${ }^{1,2^{*}}$

${ }^{1}$ Centre for Research in Agricultural Genomics (CRAG) CSIC-IRTA-UAB-UB, Edifici CRAG, Campus UAB, 08193 Bellaterra, Barcelona, Spain.

${ }^{2}$ Institut de Recerca i Tecnologia Agroalimentàries (IRTA), Edifici CRAG, Campus UAB, 08193 Bellaterra, Barcelona, Spain.

${ }^{3}$ Institut de Biologie de l'Ecole Normale Supérieure, Paris, France.

Andrea Giordano: andrea.giordano@cragenomica.es

Miguel Santo Domingo: miguel.santodomingo@cragenomica.es

Leandro Quadrana: leandro.quadrana@bio.ens.psl.eu

Marta Pujol: marta.pujol@irta.cat

Ana Montserrat Martín-Hernández: montse.martin@irta.cat

Jordi Garcia-Mas: jordi.garcia@irta.cat

${ }^{*}$ Corresponding author:

Jordi Garcia-Mas, jordi.garcia@irta.cat, Phone: +34 935636600

Andrea Giordano, andrea.giordano@cragenomica.es, Phone:+34 935636600

\section{Abstract}

Melon (Cucumis melo L.) has emerged as an alternative model to study fruit ripening due to the coexistence of climacteric and non-climacteric varieties. The previous characterization of a major QTL ETHQV8.1 sufficient to trigger climacteric ripening in a non-climacteric background allowed the identification within the QTL interval of a negative regulator of ripening CmCTR1-like (MELO3C024518), and a putative DNA demethylase CmROS1 (MELO3C024516), the orthologue of DML2, a DNA demethylase regulating fruit ripening in tomato. To understand the role of these genes in climacteric ripening, we generated homozygous CRISPR knockout mutants of CmCTR1like and CmROS1 in a climacteric genetic background. The climacteric behavior was altered in both loss-of-function mutants in two summer seasons with an advanced ethylene production profile compared to the climacteric wild type, suggesting a role of both genes in climacteric ripening in melon. Single cytosine methylome analyses of the CmROS1 knockout mutant revealed DNA methylation changes in the promoter regions of key ripening genes as ACS1, 
39 ETR1 and ACO1, and ripening associated-transcription factors as NAC-NOR,

40 RIN and CNR, suggesting the importance of CmROS1-mediated DNA

41 demethylation for triggering fruit ripening in melon.

42 Introduction

During the ripening process, fleshy fruits undergo physiological and metabolic changes affecting color, flavor, firmness, and aroma. These changes are driven by phytohormones and developmental factors and occur in a highly coordinated manner with a direct impact on fruit quality and shelf-life (Giovannoni, 2001). One of the main promoters of fruit ripening is the volatile hormone ethylene. Depending on the involvement of this hormone during ripening, fruits have been traditionally divided into i) climacteric, characterized by an increase in respiration and ethylene production at the onset of ripening and ii) non climacteric, presenting low levels of both ethylene production and respiration rate across the process (McMurchie et al., 1972). Dissecting the regulatory network underlying the control of fruit ripening has been a major goal due to its biological significance but also for its commercial value (Giovannoni et al., 2017;

56 Wang et al., 2020).

57 Important advances in the understanding of the molecular mechanisms 58 underlying climacteric fruit ripening have been made in the model species

59 tomato (Giovannoni, 2007) Ripening related mutants allowed the identification

60 of several transcription factors that are upstream regulators of ethylene 61 dependent or independent ripening. Among them RIPENING INHIBITOR (RIN),

62 NON-RIPENING (NOR), and COLORLESS NON-RIPENING (CNR)(Vrebalov et 63 al., 2002; Manning et al., 2006; Giovannoni, 2007).

64 Recent studies demonstrated that DNA methylation levels play an important 65 role at the onset of fruit ripening in tomato(Zhong et al., 2013). Modulation of 66 DNA methylation levels is governed by DNA methylases and demethylases.

67 The enzymatic removal of methylcytosine in plants is initiated by a family of 68 DNA glycosylases/lyases, including DEMETER (DME), Repressor of silencing 1 69 (ROS1), DEMETER-like2 (DML2) and DEMETER-like3 (DML3), firstly 70 characterized in the model plant Arabidopsis thaliana (Zhu, 2009). In tomato, 71 SIDML2 is induced upon the onset of ripening leading to a global DNA 72 hypomethylation during ripening (Zhong et al., 2013; Liu et al., 2015; Lang et 
73 al., 2017) Knockout using CRISPR/Cas9 system and knockdown RNAi mutants

74 in this species revealed that SIDML2 is required for normal fruit ripening by the 75 activation of ripening-induced genes and repression of several ripening76 repressed genes (Zhong et al., 2013; Lang et al., 2017). Nonetheless, the 77 tomato model is not universal as different transcriptional positive feedback circuits controlling ripening in climacteric species were identified (Lü et al., 2018).

80 Melon (Cucumis melo L.) has emerged as an alternative model to study fruit 81 ripening since both climacteric (e.g. cantalupensis types as 'Védrantais' (VED)) 82 and non-climacteric (e.g. inodorus types as 'Piel de Sapo' (PS)) genotypes 83 exist. The recent characterization of a major QTL in chromosome 8 of melon, 84 ETHQV8.1, which is sufficient to activate climacteric ripening in a non85 climacteric background, allowed the identification of candidate genes related to fruit ripening in a genomic interval of $150 \mathrm{~kb}$ that contained 14 annotated genes

87 (Pereira et al., 2020). Some of these genes are highly expressed in fruits and contain multiple non-synonymous polymorphisms distinguishing the climacteric VED from the non-climacteric PS genotype.

90 One of the candidates (CmROS1, MELO3C024516) encodes the homolog of 91 the main DNA demethylase ROS1 in Arabidopsis, which targets mainly 92 transposable element (TE) sequences and regulates some genes involved in 93 pathogen response and epidermal cell organization(Yamamuro et al., 2014; Le 94 et al., 2014). The closest orthologue in tomato, SIDML2 is crucial for the DNA 95 demethylation of fruit ripening genes including ethylene synthesis and signaling 96 (Lang et al., 2017).

97 The other candidate gene is CONSTITUTIVE TRIPLE RESPONSE 1 (CTR1) a 98 serine/threonine kinase (CmCTR1-like, MELO3C024518). This kinase interacts 99 physically with ethylene receptors as a negative regulator of the ethylene signal 100 transduction pathway(Kieber et al., 1993). In the absence of ethylene, CTR1 is 101 activated, preventing the downstream transduction pathway; when ethylene is 102 present, the ethylene receptor terminates the activation of CTR1, leading to the 103 ethylene responses (Binder, 2008). In tomato, the silencing of CTR1 promoted 104 fruit ripening, validating its role as a negative regulator of the ethylene signal 105 transduction pathway (Fu et al., 2005). 
106 In this study, we aimed to better understand the role of the two ETHQV8.1-

107 containing candidate genes CmROS1 and CmCTR1-like in fruit ripening by

108 obtaining CRISPR/Cas9-induced loss-of-function mutants in a climacteric melon

109 genotype. Furthermore, we characterized the role of CmROS1 in DNA

110 methylation homeostasis during fruit ripening.

111 Materials and methods

\section{CRISPR/Cas9 vector Construction}

113 To target CmROS1 (MELO3C024516) two different guide RNAs (gRNA) of 20

114 nucleotides in length separated by 188 bp were designed using Breaking Cas

115 tool (https://bioinfogp.cnb.csic.es/tools/breakingcas/) (Table S1). The two

116 oligonucleotides generated for each gRNA were annealed and cloned in the

117 sites Bbsl and Bsal into the plasmid ptandemgRNA. The construct was verified

118 by sequencing and then digested with Spel and Kpnl to release the cassette

119 that was then inserted into the same sites in the pB7-Cas9-TPC-polylinker

120 binary vector. Cloning vectors were gently provided by Prof. Puchta (KIT,

121 Germany).

122 For CmCTR1-like (MELO3C024518) we used the pEn-CHIMERA vector

123 provided by Prof. Puchta (KIT, Germany) to generate the entry construct. A

124 single gRNA of 20 nucleotides was designed using Breaking Cas tool

125 (https://bioinfogp.cnb.csic.es/tools/breakingcas/) (Table S1). Cloning steps of

126 the gRNA and transfer to the pDe-Cas9 binary vector were performed as

127 previously described(Schiml and Puchta, 2016).

\section{Agrobacterium mediated plant transformation}

130 Agrobacterium tumefaciens (strain AGL0) cells were transformed with the 131 binary CRISPR/Cas9 construct. Plant transformation was performed by co132 cultivation of the Agrobacterium culture with one-day-old cotyledons of VED as 133 previously described (Castelblanque et al., 2008) including the following 134 modifications: cotyledons were dissected as in (García-Almodóvar et al., 2017), 135 regeneration media was supplemented with 6-bencylaminopurine (BA) and 136 Indole-3-acetic acid (IAA) and Agrobacterium was co-cultured with the explants 
137 during a period of three days. Transgenic plants containing the bar gene were

138 selected by L-Phosphinothricin (PPT) resistance and were grown in a growth

139 room under a 12-h light/12-h dark cycle at $28^{\circ} \mathrm{C}$.

\section{Detection of mutations}

142 Genomic DNA from leaves of in vitro plantlets (T0) and from young leaves of T1 143 and T2 plants was extracted using the CTAB method with some modifications 144 as described in (Pereira et al., 2018). The transgene presence was detected by 145 PCR using specific primers targeting Cas9. Genomic regions flanking gRNA1 146 and gRNA2 of CmROS1 were amplified by PCR using specific primers. For 147 detection of mutations in CMCTR1, a region targeting the gRNA was amplified 148 with specific primers. All primers are listed in Table S2. Mutations were detected 149 by sequencing the amplified fragments and identified by double peaks in the 150 sequence chromatograms. Purified PCR products were cloned into p-Blunt II151 TOPO vector (Life Technologies) and sequencing of colonies using M13F and M13R primers was performed to confirm the mutations.

\section{Generation of T2 plants and phenotyping of climacteric ripening traits}

155 Ploidy level of T0 plants was evaluated by flow-cytometry analysis and selected

156 T0 plants for each gene were grown under greenhouse conditions (25ํㅡ for 16 157 hours and $22^{\circ} \mathrm{C}$ for 8 hours) and self-pollinated. T1 seedlings were screened for 158 the presence of Cas9 by PCR. After segregation, non-transgenic homozygous 159 edited T1 plants were selected and grown under greenhouse conditions to 160 obtain the T2 seeds for the phenotypic assay.

161 Edited T2 CmROS1 $(\mathrm{n}=8)$ and CmCTR1 plants $(\mathrm{n}=8)$ were grown randomized 162 under greenhouse conditions $\left(24^{\circ} \mathrm{C}\right.$ for 16 hours and $22^{\circ} \mathrm{C}$ for 8 hours) at 163 Caldes de Montbui (Barcelona) in 2020 and 2021. VED plants were used as a 164 wild type control plant $(n=8)$. Plants were weekly pruned and manually 165 pollinated to obtain one fruit per plant. The harvest date was determined 166 following two criteria: either abscission date, when the fruit abscised from the 
167 plant, or 5 days after the formation of the abscission layer when it was not 168 complete.

169 Ripening-related traits were evaluated as described in Pereira 2020 in two 170 consecutive summer seasons (2020 and 2021). Production of aroma (ARO), 171 chlorophyll degradation (CD) and abscission layer formation in the pedicel of 172 the fruit (ABS) were daily evaluated and firmness was measured at harvest 173 time. The visual inspection of melon fruits, attached to the plant, was performed 174 daily, from approximately 20 days after pollination (DAP) until harvest. In 175 addition, individual pictures of the fruits were obtained weekly. ARO, ABS and 176 CD were recorded as $0=$ absence and $1=$ presence. The aroma production 177 was evaluated every day by smelling the fruits. The firmness of fruit flesh was 178 measured at harvest using a penetrometer (Fruit Test ${ }^{\mathrm{TM}}$, Wagner Instruments), 179 in at least three regions of the fruit (distal, proximal and median), and the mean 180 value was registered.

\section{Ethylene production}

182 Ethylene production in planta was measured in the 2020 summer season using 183 non-invasive gas chromatography - mass spectrometry (GC-MS) method, as 184 described in (Pereira et al., 2017). The ethylene peak was monitored before 185 ripening from 20 DAP until harvest. The atmosphere of the chamber containing 186 the fruit was measured every day.

187 The ethylene peak was characterized by four traits, measured as described in 188 Pereira 2020: maximum production of ethylene in the peak (ETH), earliness of 189 ethylene production (DAPE), earliness of the ethylene peak (DAPP), and width 190 of ethylene peak (WEP).

\section{Epigenomics}

192 DNA was extracted from fruit flesh of ROS1-CRISPR-2 and the wild-type VED 193 at different ripening stages (15, 25 and 30 DAP and harvest point) following the 194 CTAB protocol(Doyle and JJ, 1990) adding a purification step using 195 Phenol:Chloroform:Isoamyl alcohol (25:24:1). For each time point, three 196 biological replicates were analysed. Bisulfite conversion, BS-seq libraries and 
197 sequencing (paired-end $100 \mathrm{nt}$ reads) were performed by BGI Tech Solutions

198 (Hong Kong). Mapping was performed on melon genome v3.6.1 (Ruggieri et 199 al., 2018) using Bismark v0.14.2 (Krueger and Andrews, 2011) and the 200 parameters: --bowtie2, -N 1, -p 3 (alignment); --ignore 5 --ignore_r2 5 -201 ignore_3prime_r2 1 (methylation extractor). Only unique mapping reads were 202 retained. The methylKit package v0.9.4 (Akalin et al., 2012) was used to 203 calculate differential methylation in 100 bp non-overlapping windows (DMRs).

204 Significance of calculated differences was determined using Fisher's exact test 205 and Benjamin-Hochberg (BH) adjustment of p-values (FDR<0.05) and 206 methylation difference cutoffs of $40 \%$ for $\mathrm{CG}, 20 \%$ for $\mathrm{CHG}$ and $20 \%$ for $\mathrm{CHH}$.

207 Differentially methylated windows within 100bp of each other were merged to 208 form larger DMRs. 100bp windows with at least six cytosines covered by a 209 minimum of six ( $\mathrm{CG}$ and $\mathrm{CHG})$ and ten $(\mathrm{CHH})$ reads per comparison were 210 considered.

\section{Statistical analyses}

213 All the statistical analyses and graphical representations were obtained using 214 the software R v3.2.3 (R Development Core Team, 2011) with the RStudio 215 v1.0.143 interface (RStudio: Integrated development environment for $R, 2012$ ).

\section{Results}

\section{Generation of CRISPR/Cas9 knockout mutants in candidate genes for ETHQV8.1 and inheritance of the editions}

To investigate the role of CmROS1 (MELO3C024516) and CmCTR1-like

224 them out using the CRISPR/Cas9 gene editing system in a climacteric genetic

225 background (VED).

226 A strategy with two target sites in exon 2 was used for CmROS1 (Fig. 1). We

227 obtained 15\% transformation efficiency, recovering in total 59 transgenic rooted

228 plants. From the transgenic plants, almost half of them (46\%) were edited.

229 Multiple independent transgenic plants were genotyped by sequencing the 230 genomic DNA spanning both target sites. Most of the editions (75\%) occurred in 231 target 1 (gRNA1) whereas only a few editions (25\%) were obtained for target 2 232 (gRNA2). Several different insertions and deletions were obtained in T0 plants 
233 with biallelic or heterozygous mutations (Fig. S1), with several plants carrying

234 the same mutation (+1 bp). A diploid biallelic line with an insertion of $1 \mathrm{bp}$ and a

235 deletion of $23 \mathrm{bp}$ that were predicted to generate truncated proteins was 236 selected for further work (Fig. 1A).

237 The selected biallelic T0 line was self-pollinated to obtain non-transgenic (Cas9

238 free) plants carrying homozygous editions. After segregation, T1 lines

239 homozygous for the $1 \mathrm{bp}$ insertion (ROS1-CRISPR-1) or the $23 \mathrm{bp}$ deletion

240 (ROS1-CRISPR-2) were selected for further study (Fig. 1A and C).

241 A different CRISPR Cas9 strategy was used to target the CmCTR1-like gene. A 242 single target site was selected in exon 6 of CmCTR1-like (Fig. 1B). For this 243 target gene we obtained $12 \%$ of transformation efficiency. Transgenic T0 plants

244 were screened for mutations in the target site and $40 \%$ were edited showing 245 mainly large or small deletions (Fig. S1). From the edited T0 plants, a biallelic 246 line carrying a 11 bp deletion and a 1 bp insertion was selected and self247 pollinated to segregate out the Cas9 transgene. The genetic editions were 248 stably transmitted to T1 plants. After segregation, a homozygous edited line 249 carrying the $11 \mathrm{bp}$ deletion (CTR1-CRISPR-1), which is predicted to generate a 250 premature termination codon, and the homozygous line with $1 \mathrm{bp}$ insertion 251 (CTR1-CRISPR-2), generating a frame shift, were grown under greenhouse 252 conditions for the characterization of fruit ripening related traits (Fig. 1B and $\mathrm{C}$ ).

Figure 1: Schematic representation of the target sites for CRISPR/Cas9 and 257 selected CRISPR edited lines. (A) Position of the gRNA target sites (red 258 triangle) and selected mutations in the CRISPR lines for CmROS1 (B) Position 259 of the gRNA target sites (red triangle) and selected mutations in the CRISPR 260 lines for CmCTR1-like (C) Fruit phenotype at harvest time of the wild type VED 261 and CRISPR edited lines.

264 CmROS1 and CmCTR1 edited plants show altered ripening phenotypes

265 ROS1-CRISPR-1/2 and CTR1-CRISPR-1/2 were evaluated and characterized 266 for ripening related traits in two consecutive summer seasons (2020 and 2021). 267 However, the line CTR1-CRISPR-2 was only characterized in 2021 due to a 268 powdery mildew infection of some replicates in 2020 that prevented its 269 evaluation. Overall, the fruit appearance (shape, weight and colour) of the 270 CRISPR edited lines did not show major differences with the wild-type VED at 
271 harvest time and no significant changes were detected in the flesh firmness

272 (Fig. 1C, Table S3). To better characterize the ripening process, we measured

273 ethylene production in planta in 2020 with a non-invasive methodology allowing

274 observing the phenotype of the downstream effects of this hormone.

275 The phenotypic characterization revealed a significant earliness of the

276 climacteric symptoms for all the edited lines showing the same ripening

277 behaviour pattern in both years (Fig. 2, Table S3). In 2020, the earliest

278 climacteric symptom was sweet aroma production (EARO), which in the

279 CRISPR edited lines for both genes appeared around two days before VED.

280 The initiation of the rind color change, which is attributed to chlorophyll

281 degradation (ECD), was appreciated almost simultaneously with the detection

282 of the abscission layer formation (EALF) and both ripening-related traits arose

283 in both CmROS1 edited lines two days before VED. The CTR1-CRISPR-1

284 edited line exhibited the earliness of the ripening related traits all at the same

285 time, which differed significantly from VED, arising around three days before

286 than VED for ECD and EALF and two days for EARO.

287 During the second summer season, we evaluated all the CRISPR edited lines.

288 In general, the environmental conditions delayed ripening of both VED and 289 mutant plants (around 4-5 days later in 2021). Despite this environmental effect,

290 all CRISPR edited lines displayed significant advances of about three days in

291 the ripening-related traits ECD, EARO and EALF when compared to VED (Fig.

292 2). Moreover, during this year, the line CTR1-CRISPR-2 was evaluated, and

293 the dataset showed the same behaviour for both CTR1 edited lines. ROS1

294 edited lines also showed the same pattern between them.

295 We also monitored fruit ethylene emission daily in 2020 without altering the 296 ripening process (Fig. 2C). The CRISPR edited lines showed a different 297 ethylene production pattern compared to wild-type VED, with both CmROS1 298 edited lines showing the same profile. In CmROS1 mutant lines, ethylene 299 production started two days before the wild-type VED and with an increment of 3002.7 to 3-fold of ethylene production (Fig. 2C and Table S3).

301 For CmCTR1 edited lines, ethylene measurements for CTR1-CRISPR-2 were 302 not available due to the infection with powdery mildew of some of the replicates 303 of this line at around 20 DAP. The CTR1-CRISPR1 line showed a significant 304 difference in the earliness of ethylene production (DAPE) and earliness of 
305 ethylene peak (DAPP). In this line, ethylene was detected around three days in

306 advance of wild-type VED. Similarly, the peak of ethylene production was also

307 advanced three days in CTR1-CRISPR1 compared to wild-type VED, however

308 this advancement was not accompanied by a significant difference in the

309 maximum quantity of ethylene produced (Fig. 2C and Table S3). Overall, these

310 results demonstrate that both candidate genes are involved in melon fruit

311 ripening.

Figure 2: Evaluation of climacteric ripening associated traits in CRISPR edited lines and VED (in two consecutive years) and ethylene emission rates. (A) Earliness of chlorophyll degradation (ECD), Earliness of production of aroma

316 (EARO) and Earliness of abscission layer formation (EALF) in 2020 (B) ECD,

317 EARO and EALF in 2021 (C) Ethylene production in attached fruits from 25 318 days after pollination (DAP) until harvest in 2020.

\section{Characterization of the ROS1-CRISPR and VED methylome at different fruit ripening stages}

323 To better understand at the molecular level the role of CmROS1 in DNA 324 demethylation and fruit ripening in melon, we generated single-cytosine 325 resolution methylomes by whole genome bisulfite sequencing from fruits of

326 ROS1-CRISPR-2 and the wild-type VED plants at 15, 25 and 30 DAP as well as 327 at harvest $(\mathrm{H})$ point (Fig. 3A).

328 When comparing the global methylation level along ripening in VED, we found 329 that methylation at CG and CHG contexts declines along fruit ripening, showing 330 around 2,000 and 4,000 hypomethylated regions (DMR) respectively in the $C G$ 331 and $\mathrm{CHG}$ context at harvest time compared to the first stage of ripening (i.e. $\mathrm{H}$ 332 vs 15 DAP) (Fig. 3B). Interestingly, these changes were more often associated 333 with promoter and intergenic regions (Fig. 3D).

334 In order to evaluate the role of ROS1 in the observed DNA methylation 335 dynamics, we compared the methylation level in the three contexts of ROS1336 CRISPR-2 and the wild-type VED plants at the same ripening stage (Fig. 3C). In 337 this way, we identified numerous changes in DNA methylation levels for the 338 three sequence contexts. In total (CG, $\mathrm{CHG}$ and $\mathrm{CHH}$ context together), we 339 found 16,968 hypermethylated DMRs at 15 DAP, 26,497 at 25 DAP, 19,928 at 34030 DAP and 43,156 at $\mathrm{H}$ time relative to VED. Overall, CRISPR-ROS1 line is 
341 associated with hypomethylation of CG and hypermethylation of CHG DMRs 342 (Fig. 3C).

343 To further investigate the targets of ROS1 we focused on the hypermethylated

344 DMRs in the CRISPR-ROS1 line (Table S4). Moreover, in $\mathrm{CHH}$ context at $\mathrm{H}$

345 time there are changes in the number of DMRs annotation between VED and

346 the edited line. Among the $\mathrm{CHH}$ hypermethylated regions in CRISPR-ROS1

347 compared to VED at $\mathrm{H}$ time, $14 \%$ are associated with TEs, $46 \%$ with intergenic,

$3486 \%$ in promoter regions (defined as $1 \mathrm{~kb}$ upstream transcriptional start sites),

349 and 33\% in genic regions (Fig. 3D).

351 Figure 3: General methylation and DMR regions at different ripening stages of

352 VED and CRISPR-ROS1 line. (A) Fruit ripening stages (B) number of DMRs

353 along ripening in VED (C) number of DMRs in VED vs CRISPR-ROS1 at the

354 same time point of ripening (D) DMRs annotation in VED along ripening (E)

355 DMRs annotation in VED vs CRISPR-ROS1 at the same time point of ripening

Notably, Gene ontology (GO) enrichment analysis of genes associated with

358 hypermethylated DMRs at $\mathrm{H}$ time in the CRISPR-ROS line compared to VED

359 and hypomethylated along ripening in VED context revealed an

360 overrepresentation of genes related to response to stress in CRISPR-ROS1

361 compared to VED (Table S5).

\section{CmROS1 targets promoter regions of key genes involved in ripening}

364 We have further analysed the methylation level of key genes known to 365 participate in the ripening process in the three contexts. Changes were found at 366 different stages of ripening in the promoter region of genes involved in the 367 ethylene biosynthesis or signaling pathway: ACS1 (MELO3C016340.2), ETR1 368 (MELO3C003906.2) and ACO1 (MELO3C014437) as well as in ripening 369 associated-transcription factors: NAC-NOR (MELO3C016540), RIN

370 (MELO3C026300.2) and CNR (MELO3C002618.2) (Fig. 4).

371 Notably, the promoter region of ACS1 appeared hypomethylated on the three 372 sequence contexts in the CRISPR-ROS1 line compared to VED in all the time 373 points studied along ripening. Furthermore, hypomethylation of the ACO1 374 promoter (CG and $\mathrm{CHH}$ context) was observed at 25 and 30 DAP and the ETR1 
375 promoter region ( $\mathrm{CHG}$ and $\mathrm{CHH}$ context) at 30 DAP. In contrast, $\mathrm{CHG}$

376 hypermethylation of NAC-NOR was found from the earliest stage until 30 DAP

377 in the mutant and hypomethylated at $30 \mathrm{DAP}$ in the $\mathrm{CHH}$ context. For the other

378 two transcription factors, we observed $\mathrm{CHH}$ hypomethylation of $\mathrm{RIN}$ and $\mathrm{CNR}$

379 promoter regions at $\mathrm{H}$ time. In combination, these results provide strong

380 evidence that CmROS1 modulates DNA methylation levels of ripening genes in

381 melon, which may have major consequences for fruit ripening.

Figure 4: DNA methylation levels of ethylene related genes and ripening 384 associated transcription factors for VED and CRISPR-ROS1 at different fruit 385 ripening development stages in the three contexts (A) $A C O 1$ in $\mathrm{CHH}$ context at 38625 (left) and 30 DAP (right) (B) ACS1 in CHG context at 25 (left) and 30 DAP 387 (right) (C) ETR1 in CHG context at 30 DAP (D) ripening associated transcription 388 factors in $\mathrm{CHH}$ context: NAC-NOR at $30 \mathrm{DAP}, \mathrm{RIN}$ at $\mathrm{H}$ point and $C N N$ at $\mathrm{H}$ 389 point.

390 Discussion

391 Advances in genome editing have been obtained applying the CRISPR/Cas 392 technology in several plant species. However, among the Cucubitaceae family 393 studies were only reported in watermelon for herbicide resistance (Tian et al., 394 2016, 2018) and cucumber for virus resistance (Chandrasekaran et al., 2016).

395 More recently, edited plantlets with a disruption of a visual reporter gene 396 (CmPDS), which could not be carried to the next generation, were generated in 397 melon using CRISPR/Cas9 (Hooghvorst et al., 2019). To our knowledge, 398 hereby we report for the first time the generation of melon knockout mutants for 399 an agronomic important trait such as fruit ripening and the inheritance of the 400 introduced mutations to the following generations using CRISPR/Cas9.

401 Melon is considered a recalcitrant species for genetic transformation. In this 402 study, we obtained on average 15\% transgenic plants and from these, $40 \%$ and $40346 \%$ were successfully edited plants for our target genes CmROS1 and 404 CmCTR1 using two or one gRNA strategy, respectively. The edited plants 405 carried several types of editions nearby the protospacer adjacent motif (PAM) 406 sequence of the target gRNA. As reported for other species (Feng et al., 2014), 407 biallelic edited plants were obtained (70\% of the edited plants), suggesting early 408 editions during developmental stages. 
409 In accordance with the mutations induced by Non-homologous end Joining

410 pathway, the sequence analysis of the edited lines revealed that the most

411 frequent editions were insertions and deletions with more than one independent

412 event exhibiting the same edition. All the gRNA used here successfully induced

413 mutations in the target genes. However, editions in $\mathrm{CmROS1}$ were mainly

414 obtained in gRNA1 suggesting a higher edition efficiency for this gRNA. In

415 addition, in contrast to the observations reported by Hooghvorst et. al., base

416 pair substitutions were not obtained for any of the genes targeted in this study.

417 Improving fruit quality and shelf life has been one of the main challenges for

418 agriculture. During the last decades, advances in understanding the ripening

419 process were approached by conventional breeding and genetic engineering

420 tools. For instance, CRISPR knockout mutants in tomato have proved the

421 importance of master ripening regulator genes(Ito et al., 2015).

422 More recent studies showed that epigenetic regulation plays a key role in fruit

423 ripening (Lü et al., 2018). The balance of global DNA methylation/demethylation

424 is altered during fruit ripening and these alterations are governed by DNA

425 demethylases. In tomato, more than 200 promoters of ripening-related genes,

426 including master regulators, ethylene related genes, fruit softening, and

427 carotenoids synthesis genes, are regulated by DNA demethylation at the onset

428 of ripening (Zhong et al., 2013).

429 In Arabidopsis, the protein repressor of silencing 1 (ROS1), which belongs to

430 the subfamily of bifunctional 5-methylcytosine DNA glycosylases/lyases, has

431 been characterized as the main sporophytic DNA demethylase(Gong et al.,

432 2002). In tomato, there are four genes (SIDML1, SIDML2, SIDML3 and SIDML4)

433 encoding putative DNA demethylases, being SIDML2 the closest ortholog to

434 Arabidopsis ROS1 gene. Furthermore, SIDML2 expression is highly correlated

435 with fruit ripening (Zhong et al., 2013; Liu et al., 2015). In melon, we have

436 identified four putative ROS1 homologues (MELO3C024516, MELO3C021451,

437 MELO3C002241 and MELO3C009432) (Fig S2). The gene MELO3C024516

438 locates in the previously identified ripening QTL interval ETHQV8.1 and

439 therefore was edited in this study. The CRISPR-ROS1/2 lines, carrying loss-of-

440 function homozygous alleles of MELO3C024516, showed an advance in

441 climacteric ripening compared to the wild type, suggesting a role of this gene in

442 the complex regulation of climacteric ripening in melon. Interestingly, RNA-seq 
443 expression analysis of several fruit ripening stages in wild type climacteric VED

444 shows that the four putative ROS1 genes have a similar expression profile

445 along ripening (Fig. S3), suggesting that more than one DNA demethylase may

446 be involved in this process. Unlike in tomato, in which the CRISPR SIDML2

447 mutant showed an inhibitory effect on fruit ripening (Lang et al., 2017), the

448 CmROS1 knockout melon fruit ripens ahead of the wild type VED. Our

449 methylome analysis of the climacteric variety VED showed an overall

450 demethylation in $\mathrm{CG}$ and $\mathrm{CHG}$ context along fruit ripening, similar to what has

451 been reported in tomato (Liu et al., 2015; Lang et al., 2017), orange (Huang et

452 al., 2019) and strawberry(Cheng et al., 2018). Furthermore, hypomethylation

453 levels in the promoter regions of key ripening genes (e.g. ACS1, ETR1, ACO1)

454 are in agreement with the phenotype displayed by the CmROS1 CRISPR lines.

455 Also, genes related to biotic stress response were also found to be

456 hypomethylated in ROS1 vs VED at harvest, suggesting a possible role of this

457 DNA demethylase in stress-response genes, as reported for ROS1, DML2,

458 DML3 in response to biotic stress in Arabidopsis (Le et al., 2014; Halter et al., 459 2021).

460 Both mutant lines of CTR1-CRISPR promote fruit ripening in melon in 461 agreement to the phenotype described when silencing LeCTR1 in tomato fruits 462 (Fu et al., 2005) and the previously described role of CTR1 as a negative 463 regulator of ethylene signaling in other species (Binder, 2008). This second 464 candidate gene of the QTL ETHQV8.1 plays also an important role in the 465 ripening process as a negative regulator affecting the initiation of the ripening 466 process but without affecting other important traits such as firmness.

467 To our knowledge, this is the first time that the CRISPR technology has been 468 implemented on genes involved in agronomically important traits in melon. The 469 implementation of this technology in this species and the inheritance of the 470 editions to the following generations is of high interest and a valuable resource 471 not only for researchers but also for breeders. We have functionally validated 472 two genes involved in the complex regulation of fruit ripening and studied in 473 depth the role of the DNA demethylase ROS1 in fruit ripening. However, as 474 mutants for both candidate genes CmROS1 and CmCTR1-like showed an 
475 altered ripening phenotype, further studies are needed to identify which of them

476 is the candidate for ETHQV8.1.

477

478 Acknowledgements

479 We acknowledge F. Garcia for technical support. This work was supported by

480 the Spanish Ministry of Economy and Competitiveness Grant RTI2018-097665-

481 B-C2, the Spanish Ministry of Science and Innovation-State Research Agency

482 (AEI) Severo Ochoa Programme for Centres of Excellence in R\&D

483 CEX2019-000902 and the CERCA Programme/Generalitat de Catalunya to

484 J.G.-M. AG was supported by the European Union's Horizon 2020 research and

485 innovation programme under Marie Skłodowska-Curie grant agreement No

486 793090. MSD was supported by a FPI grant from the Spanish Ministry of

487 Economy and Competitiveness. Work in the Quadrana group is supported by

488 the European Research Council (ERC) under the European Union's Horizon

4892020 research and innovation program (grant agreement No. 948674).

\section{References}

Akalin A, Kormaksson M, Li S, Garrett-Bakelman FE, Figueroa ME, Melnick A, Mason CE. 2012. MethylKit: a comprehensive R package for the analysis of genomewide DNA methylation profiles. Genome Biology 13, 1-9.

Binder BM. 2008. The ethylene receptors: Complex perception for a simple gas. Plant Science 175, 8-17.

Castelblanque L, Marfa V, Claveria E, I M, L P-G, Dolcet-sanjuan R. 2008. Improving the genetic transformation efficiency of Cucumis melo subsp . melo "Piel de Sapo" via Agrobacterium. Transformation, 627-632.

Chandrasekaran J, Brumin M, Wolf D, Leibman D, Klap C, Pearlsman M, Sherman A, Arazi T, Gal-On A. 2016. Development of broad virus resistance in nontransgenic cucumber using CRISPR/Cas9 technology. Molecular Plant Pathology, 1-14. Cheng J, Niu Q, Zhang B, Chen K, Yang R, Zhu JK, Zhang Y, Lang Z. 2018. Downregulation of RdDM during strawberry fruit ripening. Genome Biology 19.

Doyle, JJ. 1990. Isolation of plant DNA from fresh tissue. Focus 12, 13-15.

Feng Z, Mao Y, Xu N, et al. 2014. Multigeneration analysis reveals the inheritance, specificity, and patterns of CRISPR/Cas-induced gene modifications in Arabidopsis. Proceedings of the National Academy of Sciences 111, 4632-4637.

Fu DQ, Zhu BZ, Zhu HL, Jiang WB, Luo YB. 2005. Virus-induced gene silencing in tomato fruit. Plant Journal 43, 299-308.

García-Almodóvar RC, Gosalvez B, Aranda MA, Burgos L. 2017. Production of transgenic diploid Cucumis melo plants. Plant Cell, Tissue and Organ Culture (PCTOC) 2017 130:2 130, 323-333.

Giovannoni J. 2001. MOLECULAR BIOLOGY OF FRUIT MATURATION AND RIPENING. Annual review of plant physiology and plant molecular biology 52, 725749 . 
Giovannoni JJ. 2007. Fruit ripening mutants yield insights into ripening control.

Current Opinion in Plant Biology 10, 283-289.

Giovannoni J, Nguyen C, Ampofo B, Zhong S, Fei Z. 2017. The Epigenome and Transcriptional Dynamics of Fruit Ripening. https://doi.org/10.1146/annurev-arplant042916-040906 68, 61-84.

Gong Z, Morales-Ruiz T, Ariza RR, Roldán-Arjona T, David L, Zhu J-K. 2002. ROS1, a Repressor of Transcriptional Gene Silencing in Arabidopsis, Encodes a DNA Glycosylase/Lyase. Cell 111, 803-814.

Halter T, Wang J, Amesefe D, Lastrucci E, Charvin M, Rastogi MS, Navarro L. 2021. The arabidopsis active demethylase ros 1 cis-regulates defense genes by erasing dna methylation at promoter-regulatory regions. eLife 10, 1-62.

Hooghvorst I, López-Cristoffanini C, Nogués S. 2019. Efficient knockout of phytoene desaturase gene using CRISPR/Cas9 in melon. Scientific Reports 2019 9:1 9, $1-7$.

Huang H, Liu R, Niu Q, Tang K, Zhang B, Zhang H, Chen K, Zhu JK, Lang Z. 2019. Global increase in DNA methylation during orange fruit development and ripening. Proceedings of the National Academy of Sciences of the United States of America 116, 1430-1436.

Ito Y, Nishizawa-Yokoi A, Endo M, Mikami M, Toki S. 2015. CRISPR/Cas9mediated mutagenesis of the RIN locus that regulates tomato fruit ripening.

Biochemical and Biophysical Research Communications 467, 76-82.

Kieber JJ, Rothenberg M, Roman G, Feldmann KA, Ecker JR. 1993. CTR1, a negative regulator of the ethylene response pathway in arabidopsis, encodes a member of the Raf family of protein kinases. Cell 72, 427-441.

Krueger F, Andrews SR. 2011. Bismark: a flexible aligner and methylation caller for Bisulfite-Seq applications. Bioinformatics 27, 1571-1572.

Lang Z, Wang Y, Tang K, Tang D, Datsenka T, Cheng J, Zhang Y, Handa AK, Zhu JK. 2017. Critical roles of DNA demethylation in the activation of ripeninginduced genes and inhibition of ripening-repressed genes in tomato fruit. Proceedings of the National Academy of Sciences of the United States of America 114, E4511-E4519. Le T-N, Schumann U, Smith NA, et al. 2014. DNA demethylases target promoter transposable elements to positively regulate stress responsive genes in Arabidopsis. Genome Biology 2014 15:9 15, 1-18.

Liu R, How-Kit A, Stammitti L, et al. 2015. A DEMETER-like DNA demethylase governs tomato fruit ripening. Proceedings of the National Academy of Sciences of the United States of America 112, 10804-10809.

Lü P, Yu S, Zhu N, et al. 2018. Genome encode analyses reveal the basis of convergent evolution of fleshy fruit ripening. Nature Plants 4, 784-791.

Manning K, Tör M, Poole M, Hong Y, Thompson AJ, King GJ, Giovannoni JJ, Seymour GB. 2006. A naturally occurring epigenetic mutation in a gene encoding an SBP-box transcription factor inhibits tomato fruit ripening. Nature Genetics 2006 38:8 38, 948-952.

McMurchie EJ, McGlasson WB, Eaks IL. 1972. Treatment of fruit with propylene gives information about the biogenesis of ethylene. Nature 237, 235-6.

Pereira L, Pujol M, Garcia-mas J, Phillips MA. 2017. Non-invasive quantification of ethylene in attached fruit headspace at $1 \mathrm{p} . \mathrm{p}$. b . by gas chromatography - mass spectrometry. , 172-183.

Pereira L, Ruggieri V, Pérez S, Alexiou KG, Fernández M, Jahrmann T, Pujol M, Garcia-Mas J. 2018. QTL mapping of melon fruit quality traits using a high-density GBS-based genetic map. BMC Plant Biology 18, 1-17. 
Pereira L, Santo Domingo M, Ruggieri V, et al. 2020. Genetic dissection of climacteric fruit ripening in a melon population segregating for ripening behavior. Horticulture Research 7.

R Development Core Team. 2011. A Language and Environment for Statistical Computing. , 409.

RStudio: Integrated development environment for $\boldsymbol{R}$. 2012. Boston, MA, USA:

RStudio Inc.

Ruggieri V, Alexiou KG, Morata J, et al. 2018. An improved assembly and annotation of the melon (Cucumis melo L.) reference genome. Scientific reports 8 .

Schiml S, Puchta H. 2016. Revolutionizing plant biology $\square$ : multiple ways of genome engineering by CRISPR / Cas. Plant Methods, 1-9.

Tian S, Jiang L, Cui X, et al. 2018. Engineering herbicide-resistant watermelon variety through CRISPR/Cas9-mediated base-editing. Plant Cell Reports 2018 37:9 37, 13531356.

Tian S, Jiang L, Gao Q, et al. 2016. Efficient CRISPR/Cas9-based gene knockout in watermelon. Plant Cell Reports 2016 36:3 36, 399-406.

Vrebalov J, Ruezinsky D, Padmanabhan V, White R, Medrano D, Drake R,

Schuch W, Giovannoni J. 2002. A MADS-box gene necessary for fruit ripening at the tomato ripening-inhibitor (rin) locus. Science (New York, N.Y.) 296, 343-346.

Wang R, Angenent GC, Seymour G, de Maagd RA. 2020. Revisiting the Role of Master Regulators in Tomato Ripening. Trends in Plant Science 25, 291-301.

Yamamuro C, Miki D, Zheng Z, Ma J, Wang J, Yang Z, Dong J, Zhu J-K. 2014. Overproduction of stomatal lineage cells in Arabidopsis mutants defective in active DNA demethylation. Nature Communications 2014 5:1 5, 1-7.

Zhong S, Fei Z, Chen YR, et al. 2013. Single-base resolution methylomes of tomato fruit development reveal epigenome modifications associated with ripening. Nature Biotechnology 31, 154-159.

Zhu JK. 2009. Active DNA demethylation mediated by DNA glycosylases. Annual Review of Genetics 43, 143-166. 
A

ROS1 (MELO3C024516)

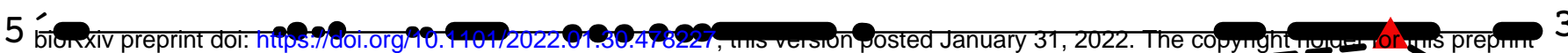

(which was not certified by peer review) is the author/funder, who has granted bioRxiv a license to display the preprint in perpetuity. dt is made available under aCC-BY-NC 4.0 Internatimentivense.

$---\overline{\text { gRNA1 }}$

AACATCTAGTCCAGGTAGTCAGGgAGCTGCCAATTCTCAAGAAAACGTGAGAACTAAAAGgAAATATGTCCGAAAGAATGCAGCCAACAAATCCCTGGAAAACCCTCTTGAACCAG GAACTCTTGATCCAGTCACCCCAGCTGGTTCTTTGGAAAATTCAAGAGGTCTAAGGACATACACTCGAAAGCGTGGCGTCAACATAATAGAAACAGGCGCATCGACTGATATGG gRNA2

VED

AACCAAGAACATCTAGTCCAGCTAGTCAGG

ROS1-CRISPR-1

AACCAAGAACATCTAGTCCAGCTATGTCAGG (+1bp)

ROS1-CRISPR-2

$A A C C$ AGG

B

CTR1-like (MELO3C024518)

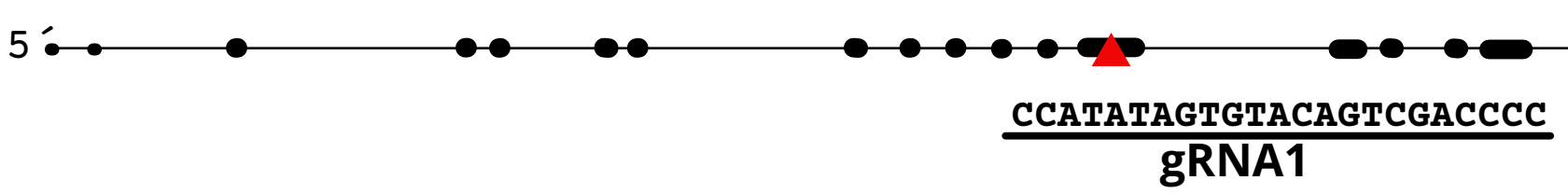

VED

TTCTCCATATAGTGTACAGTCGACCCC

CTR1-CRISPR-1

TTCT----------ACAGTCGACCCC

$(-11 \mathrm{bp})$

CTR1-CRISPR-2

TTCTCCATATAAGTGTACAGTCGACCCC (+1bp)

C

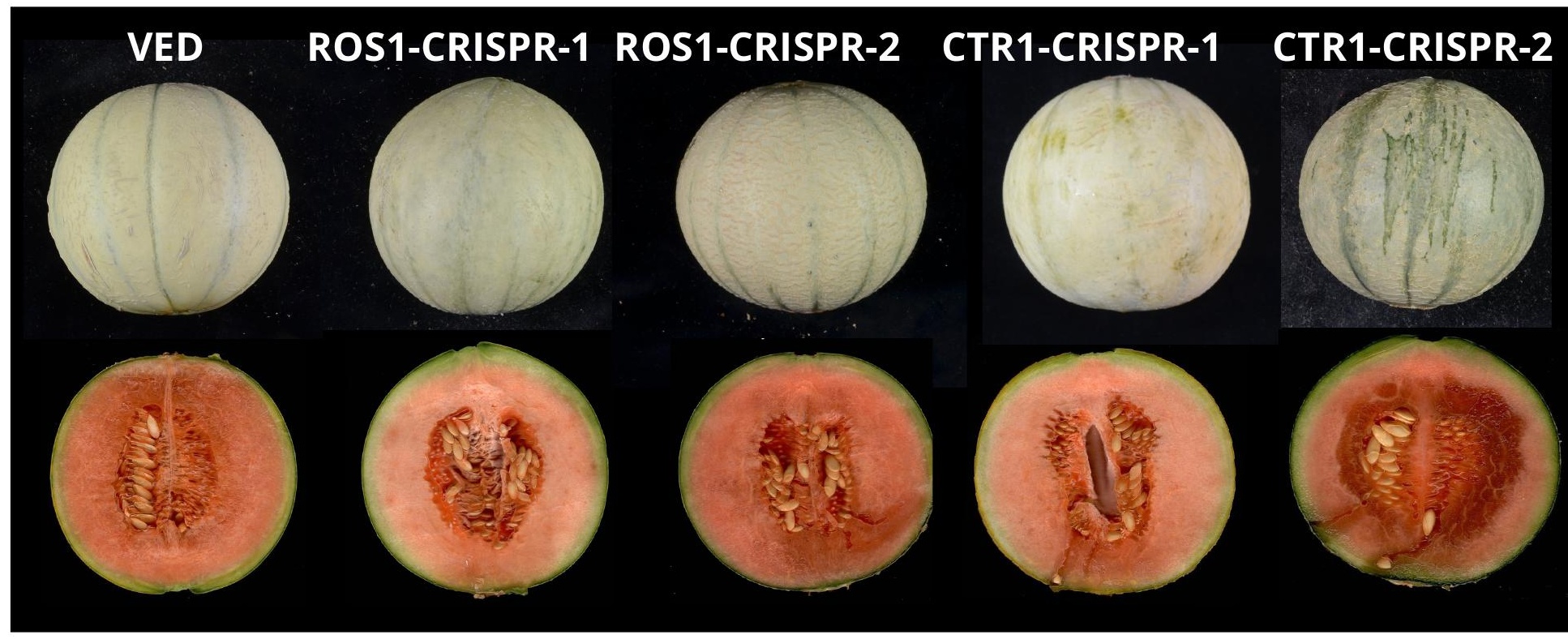




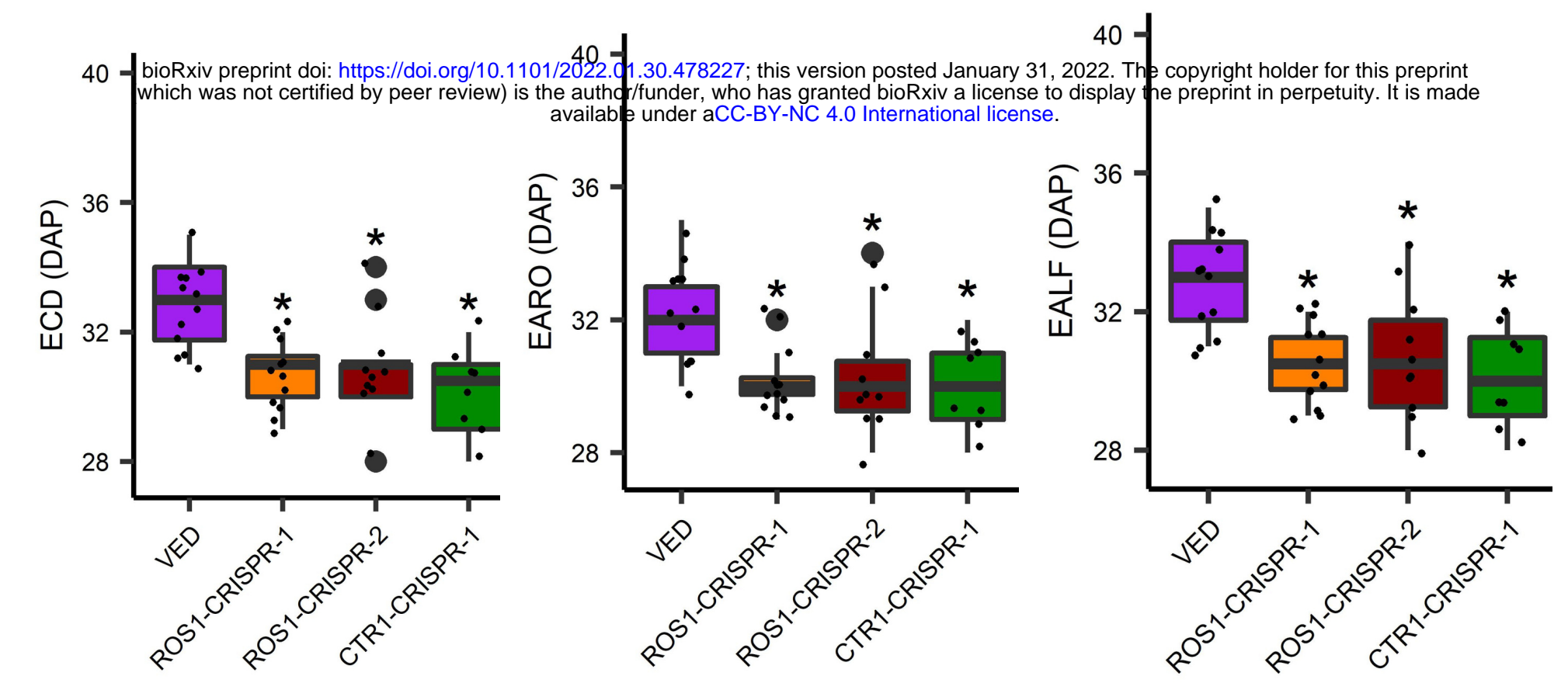

\section{Summer season 2021}
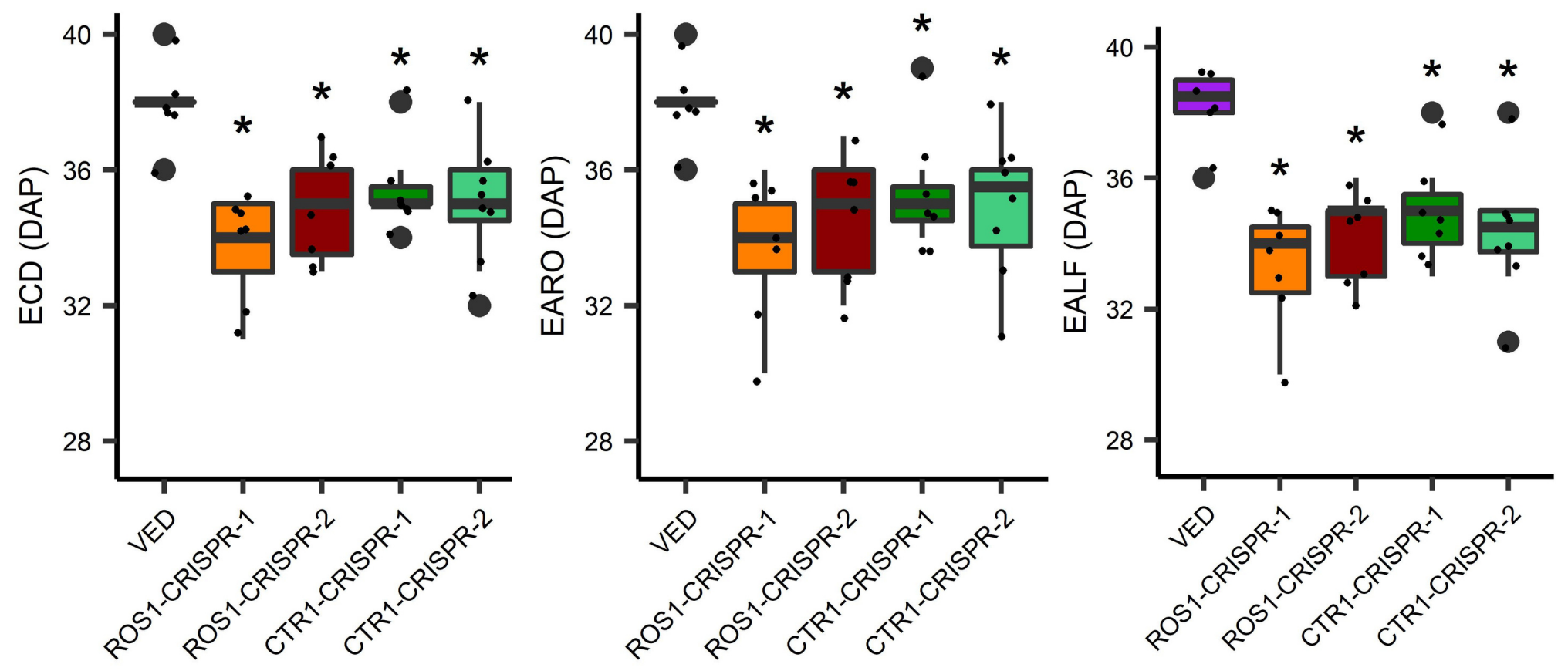

B

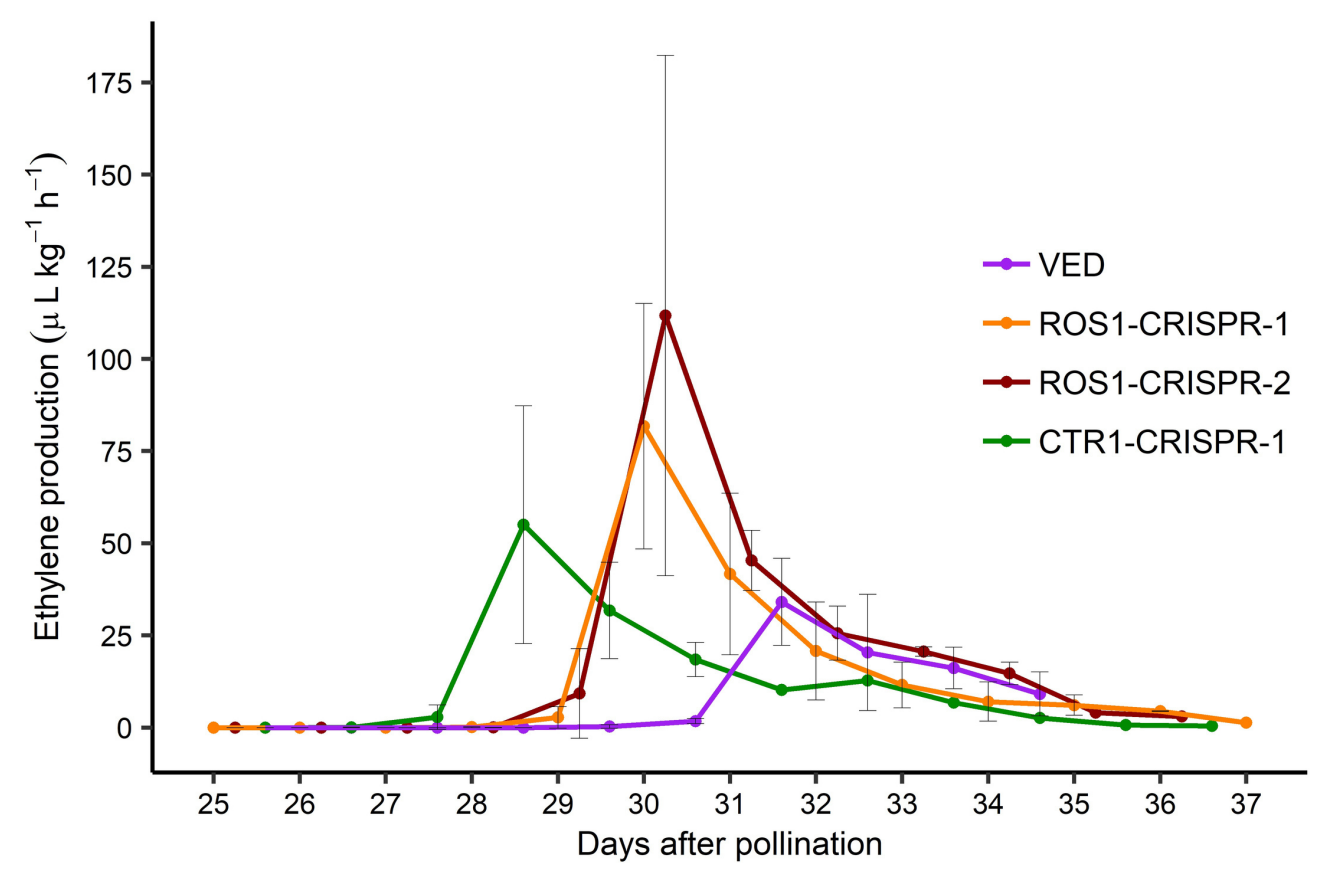




\section{Ethylene related genes}

\section{A frutt ripenting}

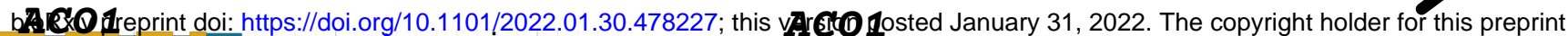

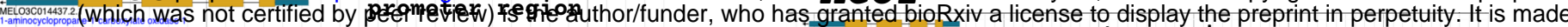
available under aCC-BYi

VED

$\mathrm{CHH}$

CRISPR-ROS 1

\section{B}

\section{FRUIT RIPENING}

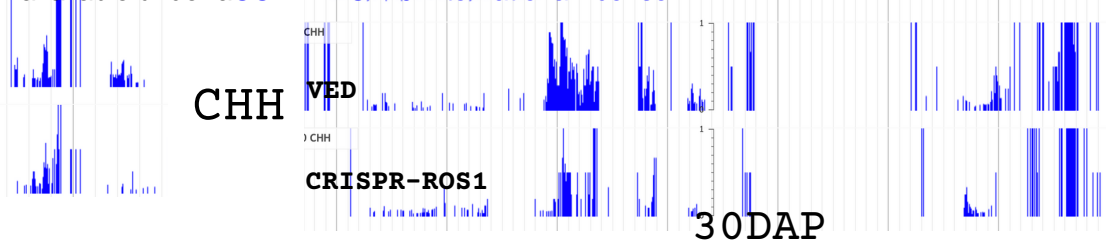

\section{ACS 1}

promoter region

MELO3C016340.2.1

$\operatorname{veD}|||||| \mid$

CHG
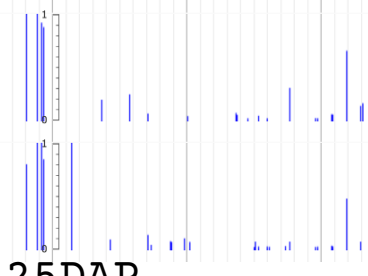

CHG

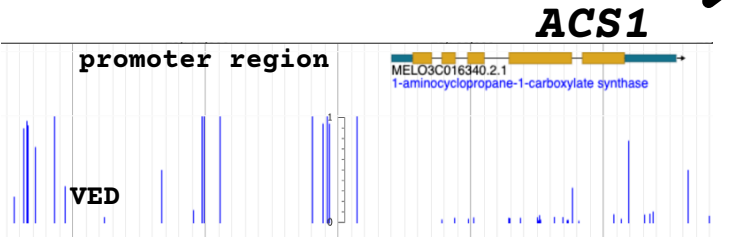

\section{C}

\section{ETR1}

$\mathrm{CHG}$

CRISPR-ROS1 promoter region

CRISPR-ROS1

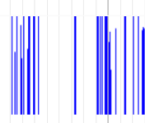

\section{Ripening associated TF}

D

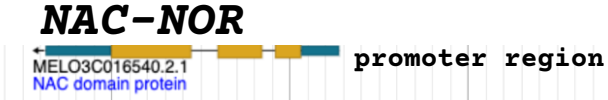

$\mathrm{CHF}$
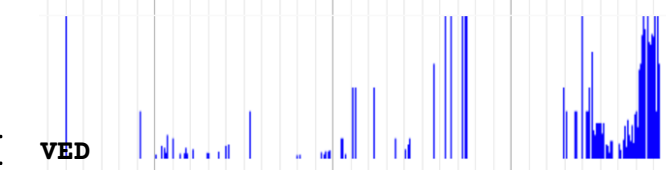

CRISPR-RのSd| ||

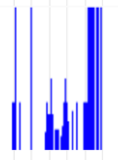

3ODAP

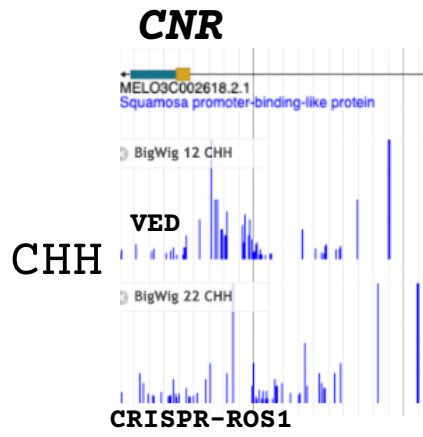

promoter region

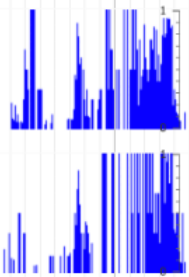

$\mathrm{H}$ point

RIN

promoter region

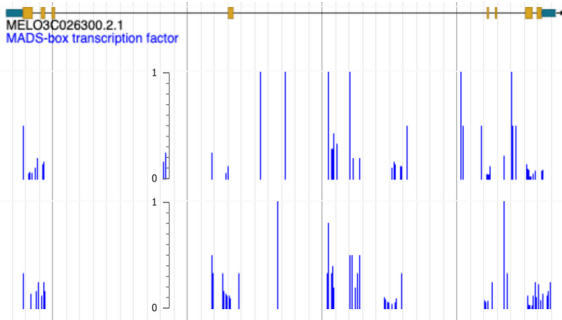

CRISPR-ROS1

$\mathrm{H}$ point 